\title{
Active room compensation for sound reinforcement using sound field separation techniques
}

Heuchel, Franz Maria; Fernandez Grande, Efren; Agerkvist, Finn T.; Shabalina, Elena

Published in:

Journal of the Acoustical Society of America

Link to article, DOI:

$10.1121 / 1.5024903$

Publication date:

2018

Document Version

Publisher's PDF, also known as Version of record

Link back to DTU Orbit

Citation (APA):

Heuchel, F. M., Fernandez Grande, E., Agerkvist, F. T., \& Shabalina, E. (2018). Active room compensation for sound reinforcement using sound field separation techniques. Journal of the Acoustical Society of America, 143(3), 1346-1354. https://doi.org/10.1121/1.5024903

\section{General rights}

Copyright and moral rights for the publications made accessible in the public portal are retained by the authors and/or other copyright owners and it is a condition of accessing publications that users recognise and abide by the legal requirements associated with these rights.

- Users may download and print one copy of any publication from the public portal for the purpose of private study or research.

- You may not further distribute the material or use it for any profit-making activity or commercial gain

- You may freely distribute the URL identifying the publication in the public portal 


\title{
Active room compensation for sound reinforcement using sound field separation techniques
}

\author{
Franz M. Heuchel, ${ }^{1, a)}$ Efren Fernandez-Grande, ${ }^{1}$ Finn T. Agerkvist, ${ }^{1}$ and Elena Shabalina ${ }^{2}$ \\ ${ }^{1}$ Acoustic Technology Group, Department of Electrical Engineering, Technical University of Denmark, \\ 2800 Kgs. Lyngby, Denmark \\ ${ }^{2} d \& b$ audiotechnik GmbH, 71522 Backnang, Germany
}

(Received 23 October 2017; revised 2 February 2018; accepted 3 February 2018; published online 6 March 2018)

\begin{abstract}
This work investigates how the sound field created by a sound reinforcement system can be controlled at low frequencies. An indoor control method is proposed which actively absorbs the sound incident on a reflecting boundary using an array of secondary sources. The sound field is separated into incident and reflected components by a microphone array close to the secondary sources, enabling the minimization of reflected components by means of optimal signals for the secondary sources. The method is purely feed-forward and assumes constant room conditions. Three different sound field separation techniques for the modeling of the reflections are investigated based on plane wave decomposition, equivalent sources, and the Spatial Fourier transform. Simulations and an experimental validation are presented, showing that the control method performs similarly well at enhancing low frequency responses with the three sound separation techniques. Resonances in the entire room are reduced, although the microphone array and secondary sources are confined to a small region close to the reflecting wall. Unlike previous control methods based on the creation of a plane wave sound field, the investigated method works in arbitrary room geometries and primary source positions. (C) 2018 Acoustical Society of America. https://doi.org/10.1121/1.5024903
\end{abstract}

[MRB]

Pages: 1346-1354

\section{INTRODUCTION}

A short reverberation time at low frequencies is crucial for the rating of venues for amplified music concerts. ${ }^{1}$ However, reverberation times at low frequencies are generally long. The sound absorption and diffusion coefficients of audiences and most acoustic treatment materials decrease with lower frequencies, as does the directivity of loudspeaker systems. This results in undesired low frequency reflections, reverberation, and mode structures.

As passive control of low frequencies can be hard to achieve, active means for reduction of reverberation and modal resonances have been the focus of research for several years and many different approaches have been developed to tackle these problems. A common approach for small rooms is to equalize the frequency response at the listening positions or zones, though this only optimizes the sound field locally, potentially creating less desirable responses outside the optimization domain. ${ }^{2-4}$ Many other works focus on active absorption where sound sources are used as sinks of acoustic energy. Some of these techniques are based on the control of the boundary impedance, e.g., the impedance of a loudspeaker membrane or a moving panel. ${ }^{5-8}$ They use pressure and velocity sensors to measure the impedance in front of the moving surface and correct it, such that the impedance matches the impedance of the incoming sound wave. This can be effective in ducts where the acoustic impedance is simple, but difficult in more general cases where the impedance is more complex. Globally effective sound control

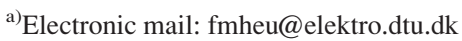

strategies have been previously investigated. ${ }^{9-13}$ These involve the creation of plane waves by a primary source array on one side of a rectangular room and their active absorption at the opposite side by a secondary array using either many loudspeakers ${ }^{9-11}$ or fewer speakers by exploiting the symmetries of a rectangular room. ${ }^{12,13}$ The latter method was coined Controlled Acoustic Bass System (CABS). ${ }^{12}$ The plane wave sound field can be regarded as being an optimal field for sound reinforcement, because of its homogeneity in space and frequency. However, the methods either require a large measurement effort ${ }^{9,10}$ or need specific placements of the primary sources. ${ }^{10,12,14}$

The current work presents a method for low frequency sound control by active absorption for situations where arbitrary, but controlled primary sources, e.g., a sound reinforcement system, create the sound. The proposed method uses sound field separation techniques ${ }^{15-18}$ on a double layer pressure microphone array to separate incoming and reflected sound field components and derive optimal filters for the secondary sources which reduce the reflected sound (thus absorbing sound by active means).

Most research on active absorption focuses on problems with unknown sound sources, thus requiring an adaptive solution. As the primary and secondary sources of this work are considered as one system, the method presented here is only feed-forward and non-adaptive. Past works on active sound absorption have already used sound field separation based on simple plane wave decomposition (PWD), ${ }^{18-20}$ but only investigated them in simulations ${ }^{20}$ or with adaptive methods for normal incidence in one-dimensional (1D) tubes. $^{18,19}$ 
The current work examines, in addition to PWD, more complex sound field separation techniques based on the equivalent source method (ESM) ${ }^{17}$ and spatial Fourier transform (SFT). ${ }^{15}$ We derive optimal compensation filters based on ESM and SFT and apply the active compensation system in complex three-dimensional sound fields in large sized rooms, both numerically and experimentally.

The remaining part of the paper is organized as follows: Sec. II introduces the mathematical formulation of the room correction system. The sound field separation techniques are described and the optimization problems, from which the optimal filters for the secondary sources are derived, formulated. A numerical study in two room geometries is presented in Sec. III. Section IV is concerned with an experimental study of the presented system. The results of the findings are discussed in Sec. V.

\section{METHODS}

The proposed active absorption method consists of an arbitrary number of primary sources, a set of $M$ secondary sources distributed over a reflecting wall, and $2 \mathrm{~N}$ pressure microphones, as sketched in Fig. 1. The secondary sources are mounted on a reflecting wall with the microphones arranged as a double layer array parallel to the $y z$-plane in front of them. The choice or number of walls covered with the secondary sources and microphones is, in principle, arbitrary. Placement along the walls involved in the most critical reflection paths is an intuitive first choice.

The purpose of the secondary sources is to actively absorb the sound radiated by the primary sources. This is achieved by driving the secondary sources with optimal signals that effectively minimize the reflections by the boundary they are mounted close to. The corresponding objective function, which shall be minimized, is quantifying the amount of reflections as measured by the microphone array and represented by the separation model. The result of the optimization is a set of $M$ complex filter coefficients which define the complex gain for each secondary source as a function of frequency.

Depending on the sound field separation method, the microphones can either measure the pressure directly (SFT) or approximate the pressure and the component of the particle velocity along the $x$ axis at the $N$ points between each pair of microphones (PWD and ESM). The pressure in

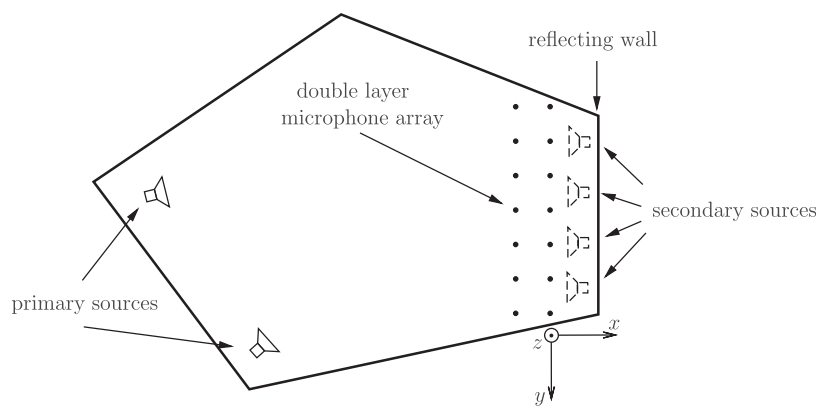

FIG. 1. Schematics of the spatial setup of the active absorption method. In the mathematical formulation the microphone array is parallel to the $y z$-plane. between can be approximated as the average pressure $p$ $=\left(p_{1}+p_{2}\right) / 2$ between the two layers separated by the distance $d$. The normal component of the particle velocity can be approximated by a finite difference approximation of Euler's equation $u_{n}=-\nabla p / \mathrm{j} \omega \rho \approx-\left(p_{2}-p_{1}\right) / \mathrm{j} \omega \rho d$.

The primary sources are assumed to be driven by a single channel such that their combined influence on the sound field can be modeled by a single transfer-function. For the application to multichannel audio reproduction, a separate set of filters per channel must be obtained and the secondary sources must be driven by the sum of the filtered signals for each channel.

In the following, the three sound separation techniques are reviewed and the respective optimal filter coefficients are derived in the frequency domain for a single frequency under the assumption that the system is linear and time-invariant. As the excitation signal is, in frequency domain, just a multiplicative constant for the pressure created by each loudspeaker, it was omitted in the equations without loss of generality.

\section{A. Plane wave decomposition}

The PWD model assumes that the sound field at each microphone position is locally composed out of two plane waves traveling in opposite directions, namely incident and reflected components,

$$
p=p_{i}+p_{r}
$$

Using the pressure $p$ and the normal particle velocity $u_{n}$, one can find the amplitudes of these components, ${ }^{19}$

$$
\begin{aligned}
& p_{i}=\frac{1}{2}\left(p+\rho c u_{n}\right), \\
& p_{r}=\frac{1}{2}\left(p-\rho c u_{n}\right) .
\end{aligned}
$$

If the boundary is totally absorptive, the reflected component will vanish.

The optimization problem is defined as follows. Let $\mathbf{p}_{p}$ $\in \mathbb{C}^{N}$ be the pressure from the primary sources to the $N$ points between the two measurement layers. Let $\mathbf{H}_{s}^{p} \in \mathbb{C}^{N \times M}$ be the pressure transfer matrix from the $M$ secondary sources to the microphone positions. The signal to each secondary source is represented by the frequency dependent filter coefficients or gains $\mathbf{w} \in \mathbb{C}^{M}$. The total pressure at the microphone positions

$$
\mathbf{p}=\mathbf{p}_{p}+\mathbf{H}_{s}^{p} \mathbf{w}
$$

is the sum of pressures by the primary and filtered secondary sources and analogously the particle velocity

$$
\mathbf{u}_{n}=\mathbf{u}_{p}+\mathbf{H}_{s}^{u} \mathbf{w} .
$$

According to Eqs. (3), (4), and (5), the reflected pressure as a function of the filters between each microphone pair is

$$
\mathbf{p}_{r}=\frac{1}{2}\left(\mathbf{p}-\rho c \mathbf{u}_{n}\right) .
$$


Minimizing $\left\|\mathbf{p}_{r}\right\|^{2}$ by an optimal choice of $\mathbf{w}$ effectively minimizes the reflections by the boundary via the absorption of sound by the secondary speakers. From Eqs. (4), (5), and (6), the optimal filter is

$$
\begin{aligned}
\mathbf{w}_{\mathrm{opt}}^{\mathrm{PWD}} & :=\underset{\mathbf{w}}{\arg \min }\left\|\mathbf{p}_{r}\right\|^{2} \\
& =\underset{\mathbf{w}}{\arg \min }\left\|\left(\mathbf{p}_{p}-\rho c \mathbf{u}_{p}\right)+\left(\mathbf{H}_{s}^{p}-\rho c \mathbf{H}_{s}^{u}\right) \mathbf{w}\right\|^{2} .
\end{aligned}
$$

This is a typical least-square problem with the solution

$$
\mathbf{w}_{\mathrm{opt}}^{\mathrm{PWD}}=-\left(\mathbf{H}_{s}^{p}-\rho c \mathbf{H}_{s}^{u}\right)^{+}\left(\mathbf{p}_{p}-\rho c \mathbf{u}_{p}\right),
$$

where the superscript + denotes the pseudo-inverse.

\section{B. Equivalent source method}

The method of equivalent sources can be used as a sound field separation technique. ${ }^{17}$ In ESM, equivalent sources are placed on two sides of the measurement surface (microphone array), as shown in Fig. 2. $N_{b}$ virtual sources on the side closer to the wall model the reflected sound field and $N_{a}$ sources on the other side model the incident sound field.

One can express the total pressure and particle velocity generated by the primary and secondary sources at the microphone positions as a function of the equivalent source strengths ${ }^{17} \mathbf{q}_{a} \in \mathbb{C}^{N_{a}}$ and $\mathbf{q}_{b} \in \mathbb{C}^{N_{b}}$,

$$
\begin{aligned}
{\left[\begin{array}{l}
\mathbf{p} \\
\mathbf{u}
\end{array}\right] } & =\left[\begin{array}{l}
\mathbf{p}_{p} \\
\mathbf{u}_{p}
\end{array}\right]+\left[\begin{array}{l}
\mathbf{H}_{s}^{p} \\
\mathbf{H}_{s}^{u}
\end{array}\right] \mathbf{w} \\
& =\underbrace{\left[\begin{array}{cc}
j \omega \rho \mathbf{G}_{h, a} & j \omega \rho \mathbf{G}_{h, b} \\
-\mathbf{G}_{h, a}^{u} & -\mathbf{G}_{h, b}^{u}
\end{array}\right]}_{:=\mathbf{G} \in \mathbb{C}^{2 M \times\left(N a+N_{b}\right)}}\left[\begin{array}{l}
\mathbf{q}_{a} \\
\mathbf{q}_{b}
\end{array}\right] .
\end{aligned}
$$

Here, $\mathbf{G}_{h, *} \in \mathbb{C}^{M \times N_{*}}$ denotes the matrix of the free-field Green's function $G\left(\mathbf{r}, \mathbf{r}^{\prime}\right)=\mathrm{e}^{-\mathrm{j} k|| \mathbf{r}-\mathbf{r}^{\prime} \|} / 4 \pi\left\|\mathbf{r}-\mathbf{r}^{\prime}\right\|$ between the microphone positions and the equivalent sources on either side, and $\mathbf{G}_{h, *}^{u} \in \mathbb{C}^{M \times N_{*}}$ denotes the matrix of derivatives of

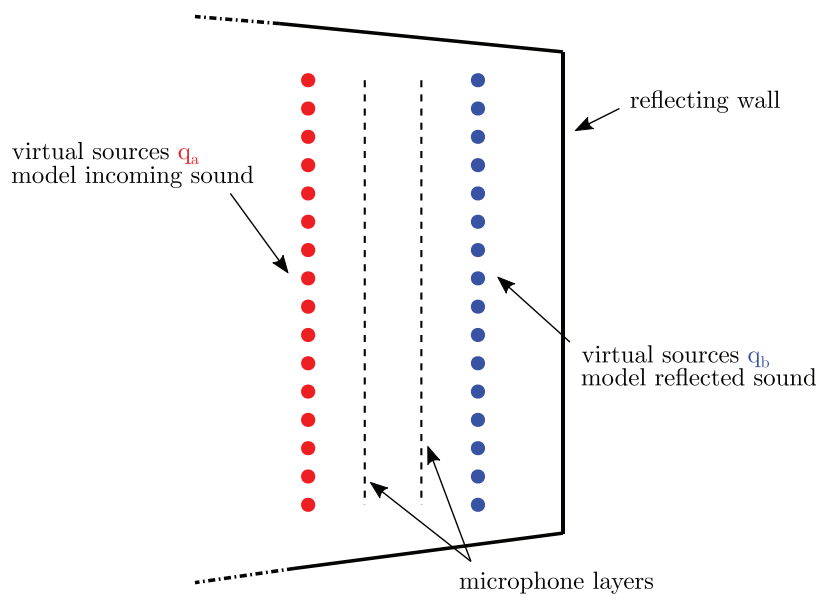

FIG. 2. (Color online) In the ESM, the sound field at the measurement planes is modeled as originating from virtual sources on both sides of the planes. the Green's function $G^{u}\left(\mathbf{r}, \mathbf{r}^{\prime}\right)=(\partial / \partial n) G\left(\mathbf{r}, \mathbf{r}^{\prime}\right)$, along the normal component of the measurement surface. By inverting Eq. (9) using the regularized pseudo-inverse $\mathbf{G}^{+}$ $\in \mathbb{C}^{\left(N_{a}+N_{b}\right) \times 2 M}$ we find the equivalent source strengths as a function of the filter coefficients. As one is only interested in the equivalent source strengths which model the reflections, $\mathbf{q}_{b}$, one takes the last $N_{b}$ rows of $\mathbf{G}^{+}$which are relevant for $\mathbf{q}_{b}$ to form a new matrix $\mathbf{G}_{\mathbf{q}_{b}}^{+} \in \mathbb{C}^{N_{b} \times 2 M}$ such that

$$
\mathbf{q}_{b}=\mathbf{G}_{\mathbf{q}_{b}}^{+}\left(\left[\begin{array}{c}
\mathbf{p}_{p} \\
\mathbf{u}_{p}
\end{array}\right]+\left[\begin{array}{c}
\mathbf{H}_{s}^{p} \\
\mathbf{H}_{s}^{u}
\end{array}\right] \mathbf{w}\right)
$$

The optimal filter

$$
\mathbf{w}_{\mathrm{opt}}^{\mathrm{ESM}}:=\underset{\mathbf{w}}{\arg \min }\left\|\mathbf{q}_{b}\right\|^{2}=-\left(\mathbf{G}_{\mathbf{q}_{b}}^{+}\left[\begin{array}{c}
\mathbf{H}_{s}^{p} \\
\mathbf{H}_{s}^{u}
\end{array}\right]\right)^{+} \mathbf{G}_{\mathbf{q}_{b}}^{+}\left[\begin{array}{l}
\mathbf{p}_{p} \\
\mathbf{u}_{p}
\end{array}\right]
$$

reduces the strengths of the equivalent sources representing the reflections and therefore, in this model, maximizes the absorption by the secondary sources.

\section{Spatial Fourier transform}

A technique based on the SFT using a pressure measurement in two parallel planes can also separate sound fields. ${ }^{15}$ This separation technique is exploited for the current problem.

Let us assume for now that the pressure is known continuously on the two parallel measurement planes $x=x_{a}$ and $x=x_{b}$ and let $P\left(k_{y}, k_{z}, x\right)$ denote the pressure in wavenumber domain obtained by a two-dimensional SFT. ${ }^{21}$ The sound field can then be decomposed along the $x$ axis into incident, $P_{i}$, and reflected, $P_{r}$, components at a third plane $x_{0}:=x_{a}$ $+d_{a}=x_{b}-d_{b}{ }^{15}$

$$
\begin{aligned}
{\left[\begin{array}{c}
P_{i}\left(k_{y}, k_{z}, x_{0}\right) \\
P_{r}\left(k_{y}, k_{z}, x_{0}\right)
\end{array}\right]=} & \frac{1}{-2 \mathrm{j} \sin \left(k_{x}\left(d_{a}+d_{b}\right)\right)} \\
& \times\left[\begin{array}{cc}
\mathrm{e}^{\mathrm{j} k_{x} d_{b}} & -\mathrm{e}^{-\mathrm{j} k_{x} d_{a}} \\
-\mathrm{e}^{-\mathrm{j} k_{x} d_{b}} & \mathrm{e}^{\mathrm{j} k_{x} d_{a}}
\end{array}\right] \\
& \times\left[\begin{array}{l}
P\left(k_{y}, k_{z}, x_{a}\right) \\
P\left(k_{y}, k_{z}, x_{b}\right)
\end{array}\right] .
\end{aligned}
$$

Note that the equation above uses the convention that a plane wave traveling in the positive $x$-direction is described by an exponential $\mathrm{e}^{-\mathrm{j} k x}$.

The total pressure field in wavenumber domain $P\left(k_{y}, k_{z}, x, \mathbf{w}\right)$ is the sum of the transformed pressures $P_{p}$ due to the primary source and $\mathbf{P}_{s} \in \mathbb{C}^{M}$ due to the set of secondary sources multiplied by the corresponding filter gain $\mathbf{w}$,

$$
P\left(k_{y}, k_{z}, x, \mathbf{w}\right)=P_{p}\left(k_{y}, k_{z}, x\right)+\mathbf{P}_{s}^{T}\left(k_{y}, k_{z}, x\right) \mathbf{w} .
$$

Combining Eqs. (12) and (13), the reflected pressure component expressed by the primary and secondary sound field reads 


$$
\begin{aligned}
P_{r}\left(k_{y}, k_{z}, x_{0}, \mathbf{w}\right)= & \frac{1}{-2 \mathrm{j} \sin \left(k_{x}\left(d_{a}+d_{b}\right)\right)} \\
& \times\left(\left[P_{p}\left(k_{y}, k_{z}, x_{b}\right) \mathrm{e}^{-\mathrm{j} k_{x} d_{a}}\right.\right. \\
& \left.-P_{p}\left(k_{y}, k_{z}, x_{a}\right) \mathrm{e}^{\mathrm{j} k_{x} d_{b}}\right] \\
& +\left[\mathbf{P}_{s}^{T}\left(k_{y}, k_{z}, x_{b}\right) \mathrm{e}^{-\mathrm{j} k_{x} d_{a}}\right. \\
& \left.\left.-\mathbf{P}_{s}^{T}\left(k_{y}, k_{z}, x_{a}\right) \mathrm{e}^{\mathrm{j} k_{x} d_{b}}\right] \mathbf{w}\right) \\
= & : \alpha\left(P_{p}^{\prime}+\mathbf{P}_{s}^{\prime} \cdot \mathbf{w}\right),
\end{aligned}
$$

where $\alpha P_{p}^{\prime}$ and $\alpha \mathbf{P}_{s}^{\prime}$ represent the reflected wave field components propagating along the negative $x$-direction created by the primary and secondary sources, respectively.

The optimal filter

$$
\mathbf{w}_{\text {opt }}^{\mathrm{SFT}}:=\underset{\mathbf{w}}{\arg \min }\left(\iint_{k_{y}, k_{z} \in \mathbb{R}}\left|P_{r}\left(k_{y}, k_{z}, x_{0}, \mathbf{w}\right)\right|^{2} \mathrm{~d} k_{y} \mathrm{~d} k_{z}\right)
$$

is designed to reduce all reflected wave space components of the pressure field. To calculate $\mathbf{w}_{\mathrm{opt}}^{\mathrm{SFT}}$ the mean square pressure is written explicitly as a function of the filter gains,

$$
\begin{aligned}
\left|P_{r}\right|^{2} & =P_{r}^{H} P_{r} \\
& =\alpha^{2}\left(\mathbf{w}^{H} \mathbf{P}_{s}^{\prime H} \mathbf{P}_{s}^{\prime} \mathbf{w}+\mathbf{w}^{H} \mathbf{P}_{s}^{\prime H} P_{p}^{\prime}+P_{p}^{\prime H} \mathbf{P}_{s}^{\prime} \mathbf{w}+P_{p}^{\prime H} P_{p}^{\prime}\right),
\end{aligned}
$$

noting that the integration operation is linear. From convex quadratic minimization it can be found that the integrated form of Eq. (16) has a unique minimum at

$$
\mathbf{w}_{\mathrm{opt}}^{\mathrm{SFT}}=-\mathbf{A}^{-1} \mathbf{b},
$$

where

$$
\begin{aligned}
& \mathbf{A}:=\iint_{k_{y}, k_{z} \in \mathbb{R}} \mathbf{P}_{s}^{\prime H} \mathbf{P}_{s}^{\prime} \mathrm{d} k_{y} \mathrm{~d} k_{z}, \\
& \mathbf{b}:=\iint_{k_{y}, k_{z} \in \mathbb{R}} \mathbf{P}_{s}^{\prime H} P_{p}^{\prime} \mathrm{d} k_{y} \mathrm{~d} k_{z},
\end{aligned}
$$

if $\mathbf{A}$ is positive-definite.

In practice, the sound field can only be measured at a finite number of discrete points, so the continuous Fourier transform and integrals have to be replaced by their discrete counterparts. $^{21}$

The linear system of Eq. (17) might be badly conditioned due to its inverse nature because the evanescent wave components are included in the integration domain. Consequently, the linear system was solved using Tikhonov $L_{2}$ regularization where the regularization parameter was estimated using generalized cross validation. ${ }^{22}$

\section{Controlled Acoustic Bass System}

$\mathrm{CABS}^{12}$ has been previously proposed as a method for global sound field control at low frequencies and is used in the present study for benchmarking. In CABS a specific symmetrical positioning of primary and secondary sources on two opposite walls and a rectangular domain geometry lead, up to a certain frequency, to a simple, quasi-1D sound field consisting only of plane waves traveling in opposite directions.

This can be briefly explained as follows: for a rectangular room with rigid boundaries, sources placed on the nodal planes of a $y z$-tangential mode $(0, j, k)$ only excite the modes $(i, 2 v j, 2 u k)$ with $i, j, k, u, v \in \mathbb{N}_{0}$. [This can be shown by inspection of the pressure in terms of the rectangular room Green's function and expression of the finite sum of the excitation terms over the sources using $\sum_{k=0}^{n} \cos (a k+z)$ $=\csc (a / 2) \sin ((1 / 2) a(n+1)) \cos (a n / 2+z)$ if $a \neq 2 \pi m$, $m \in \mathbb{N}$.] In this case the sound field is dominated by $x$-axial modes $(i, 0,0)$ for frequencies below the smallest of the eigenfrequencies of modes $(0,2 j, 0)$ and $(0,0,2 k)$. This smallest eigenfrequency will be denoted by $f_{p}$ in the remainder of this work.

This one-dimensionality results in a simple filter enabling optimal active absorption by the secondary sources. The filter consists of a delay, an inversion, and a gain reduction. The delay is set to the time the sound needs to travel through the room. The gain reduction accounts for the loss of energy while the wave travels from one side of the room to the other (in the case that the walls are not perfectly rigid). Below $f_{p}$ the resulting sound field is approximately a plane wave traveling from the primary to the secondary sources.

\section{NUMERICAL RESULTS}

The active absorption method is numerically investigated in two setups resembling an application scenario in different room geometries. They are depicted in Figs. 3(a) and 3(b). The finite-element-method is used to model the sound field and to calculate the pressure transfer functions between sources and microphones.

Note that two separate sets of microphone positions are used: the optimal filters are based on measurements in two layers close to the secondary sources and the resulting sound field is evaluated at positions distributed throughout the room (not shown in Fig. 3).

\section{A. Rectangular room}

\section{Setup}

Setup 1, shown in Fig. 3(a), is a rectangular room of dimensions $12.5 \mathrm{~m} \times 12.2 \mathrm{~m} \times 3.6 \mathrm{~m}$. Four primary monopole sources with volume velocities of $1 \mathrm{~m}^{3} \mathrm{~s}^{-1}$ are placed on the nodal planes of the $(0,4,1) y z$-tangential mode at the wall at $x=0$. The 45 secondary monopole sources are arranged in a $9 \times 5$ grid on the opposite wall. These include four sources on the nodal planes of the $(0,4,1) y z$-tangential mode for the implementation of CABS with four sources per wall. Two microphones are placed 1.0 and $1.5 \mathrm{~m}$ in front of each secondary source. These form the double layer microphone array that creates the input to the filter computations. The resulting sound field is evaluated on a $11 \times 9$ grid of positions distributed throughout the whole room at a height 


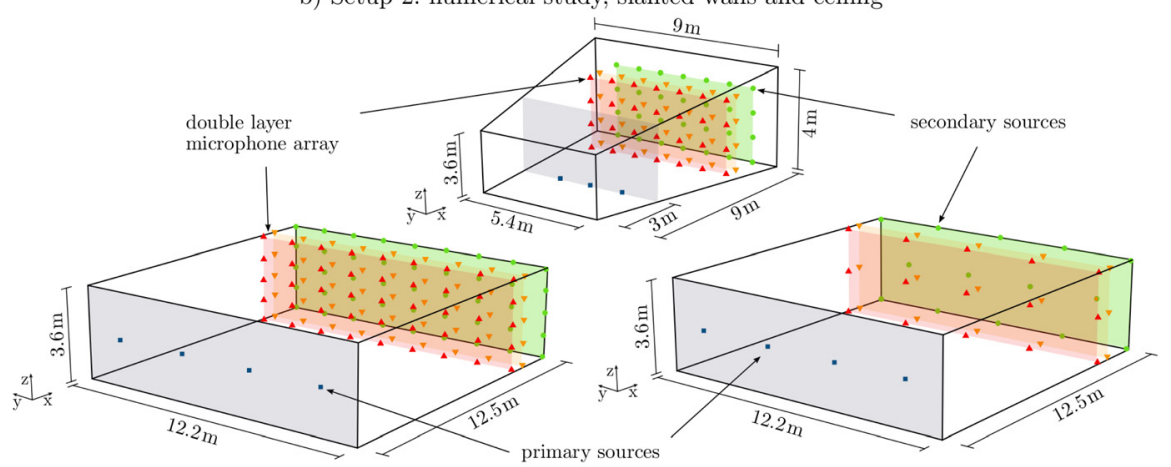

a) Setup 1: numerical study, rectangular

b) Setup 3: experiment
FIG. 3. (Color online) Setups for the numerical and experimental studies with positions of primary sources (squares), secondary sources (circles), and the two layer microphone array (up and down facing triangles). The plane containing the secondary sources is placed against the wall with a distance of 1 and $1.5 \mathrm{~m}$ to the two microphone array layers. of $1.7 \mathrm{~m}$. The impedance of the ceiling is modeled by the Miki model ${ }^{23}$ with a flow resistivity of $2 \times 10^{5} \mathrm{Ns} \mathrm{m}^{-4}$, which gives a random incidence absorption coefficient of $0.09-0.16$ in the frequency range $30-120 \mathrm{~Hz}$. All other walls have a high, but finite flow resistivity of $2 \times 10^{7} \mathrm{Ns} \mathrm{m}^{-4}$. The boundary impedances are chosen to mimic the room used for the experiment. The Nyquist frequency is $225 \mathrm{~Hz}$ given this spatial sampling, so aliasing is avoided in the frequency range of interest.

The working frequency range of CABS is limited by $f_{p}$, which is the eigenfrequency of the $z$-axial mode $(0,0,2)$, i.e., $95 \mathrm{~Hz}$. The optimal delay of $390 \mathrm{~ms}$ and gain of $-2 \mathrm{~dB}$ for CABS in this setup was found by a parameter search. ${ }^{13}$

For the ESM method, the equivalent sources were distributed over two rectangular surfaces of the same size as the microphone array on both sides of the measurement surface with a distance of $1 \mathrm{~m}$ and density of 9 equivalent sources per square meter.

\section{Result}

Figure 4 shows the resulting frequency responses in the room without control, a reference case where the back wall is implemented as a perfectly absorptive boundary using a Perfectly Matched Layer (PML), the CABS method, and the active absorption method using the three different sound separation techniques (PWD, ESM, SFT).

Without control [Fig. 4(a)], i.e., only the primary sources are active, the frequency responses show many resonances. Up to around $95 \mathrm{~Hz}$ only axial modes in the $x$-direction are excited, due to the special placement of the primary sources. In this frequency range CABS is effective [Fig. 4(c)]. A perfectly absorbing system has the same frequency responses as the PML case. It can be considered as an infinitely long duct where the mode at $95 \mathrm{~Hz}$ marks the first excited higherorder mode. This mode cannot be reduced by the absorption method as it is caused by reflections between the floor and ceiling and the sound field separation happens only in the $x$-direction. SFT, PWD, and ESM remove the room modes almost completely below $95 \mathrm{~Hz}$. The control method based on PWD, ESM, and SFT perform similar to CABS in that region. The linear phase in the room center shows that the sound field is similar to a plane-wave. Above $95 \mathrm{~Hz}$ the sound field is a combination of axial, transversal, and oblique modes. While CABS is not able to damp resonances in this region, the active absorption based on sound field separation techniques (PWD, ESM, SFT) produces results that are close to the PML case and therefore lead to nearly full absorption in the investigated frequency range.

\section{B. Room with slanted walls and ceiling}

\section{Setup}

The domain of setup 2 [Fig. 3(b)] is a room with slanted ceiling and side walls. Three primary sources are placed on the floor in some distance to the front wall. The secondary sources and double layer microphone array are arranged in a $7 \times 4$ grid. Again, the dense sampling will avoid any aliasing effects in the frequency range of interest. All walls have a high, but finite flow resistivity of $2 \times 10^{7} \mathrm{Ns} \mathrm{m}^{-4}$. The placement of sources and the room geometry break the assumptions on which CABS is based, leading to a performance of CABS similar to the uncontrolled case (not shown). In comparison to the rectangular setup 1, this geometry represents a more arbitrary situation with a more complex sound field.

\section{Result}

Figure 5 compares the active absorption method using the different separation techniques with the reference case where the back wall is a PML layer and a case without control by secondary sources. The absorption system with PWD, ESM, or SFT separation clearly reduces the room resonances in comparison to the case without control, as seen by less variation in mean sound pressure level (SPL) and a lower standard deviation compared to the uncontrolled case. The controlled sound field is more homogeneous in both space and frequency. Both mean and standard deviation of the PWD separation technique closely follow the result for the PML case, suggesting nearly total absorption of the incident sound. The ESM deviates from the reference case in the $110-120 \mathrm{~Hz}$ range with higher mean SPL and lower standard deviation. The SFT technique performs worst, but still leads to a more homogeneous sound field in comparison to the uncontrolled case (see discussion in Sec. V). 

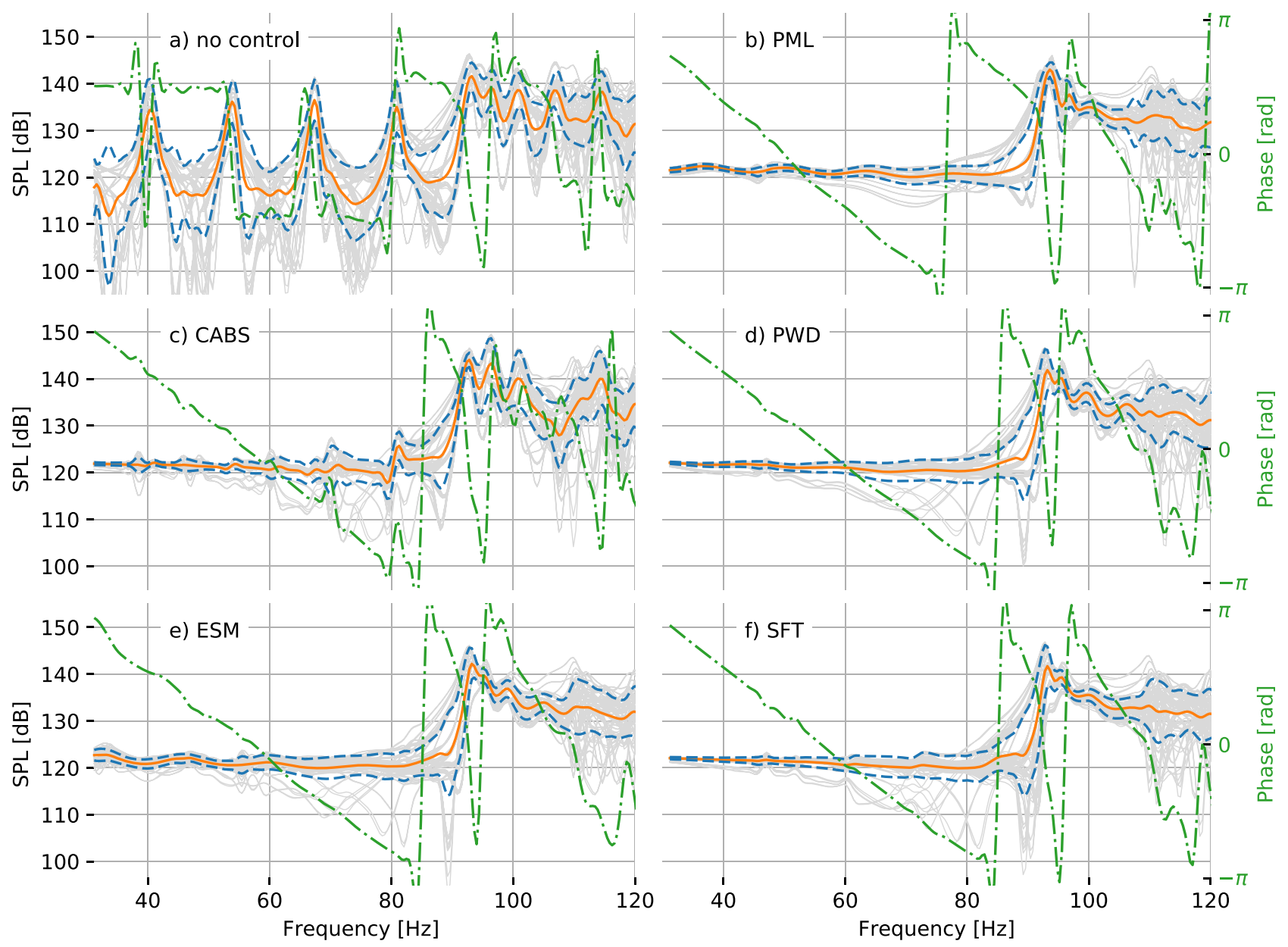

FIG. 4. (Color online) Simulated room frequency responses for the different control techniques in the rectangular room (setup 1). Single magnitude responses (thin line), their average (continuous line), and standard deviation (dashed line). The phase response is given for one position in the center of the room (dashed-dotted line).

\section{EXPERIMENTAL STUDY}

A sketch and picture of the experimental setup are shown in Figs. 3(c) and 6, respectively. The room has the same dimensions and placement of primary loudspeakers as setup 1 of the numerical study. The ceiling has been acoustically treated by $10 \mathrm{~cm}$ thick Basotect plates suspended by $10 \mathrm{~cm}$ and covering around one-half of the ceiling area. The other surfaces of the room are approximately sound hard. Fourteen secondary loudspeakers are distributed over the back wall including 4 sources at nodal planes of the $y z$-tangential mode $(0,4,1)$. This again enables the investigation of CABS with the same setup. The double layer microphone array is arranged in a $5 \times 3$ grid with a distance of 1.5 and $1.0 \mathrm{~m}$ to the back wall. The sparse sampling leads to Nyquist frequencies of 56 and $95 \mathrm{~Hz}$ along the $y$ and $z$ axes, respectively. The resulting sound field in the room was evaluated at 16 positions distributed equally around the room at a height of $1.7 \mathrm{~m}$.

The measurement was conducted as follows. First, the transfer-functions from all sources to all microphone positions were measured. Then, the responses to the double layer microphone array close to the secondary sources were used to calculate the filter coefficients for the three sound field separation techniques (PWD, ESM, SFT). Last, the resulting sound pressure in the room was found by linear combination of the contributions from the primary sound sources and the filtered secondary sources.

CABS was also implemented for comparison. The delay and gain parameters were estimated by combining the separately measured frequency responses from primary and secondary sources for a range of different delay and gain values and finding the solution with the lowest variation in frequency and space. ${ }^{13}$ This resulted in a gain of $-2 \mathrm{~dB}$ and a time delay of $390 \mathrm{~ms}$ of the secondary sources.

Figure 7 shows the resulting frequency responses without control, with CABS, and with the proposed absorption system using the three separation techniques (PWD, ESM, SFT). Without control [Fig. 7(a)], one can observe three distinct resonances at 42,55 , and $68 \mathrm{~Hz}$, which correspond to the axial modes along the $x$-direction, and a more complex sound field with more resonances above $70 \mathrm{~Hz}$.

CABS is only able to compensate for the first prominent mode. The poor performance can most likely be attributed to 


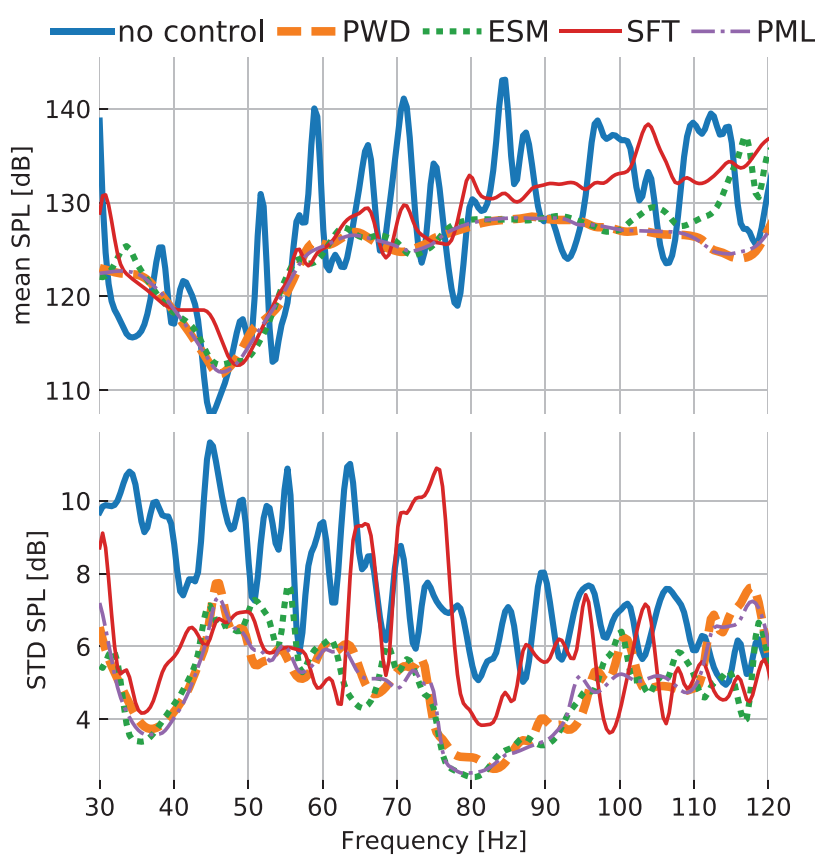

FIG. 5. (Color online) Simulated frequency responses for the different control techniques in the room with slanted walls (setup 2). Top: spatial average of magnitude response. Bottom: spatial standard deviation.

the large size of the room and its deviations from a perfectly rectangular room (see Sec. V).

In contrast, the method presented in this paper based on PWD, ESM, and SFT spatially homogenizes the sound field and effectively damps room resonances up to around $70 \mathrm{~Hz}$ [Figs. 7(c)-7(e)]. The linearized phase in the center of the room shows that the sound field is similar to a plane wave. The three sound field separation techniques lead to similar results with only minor differences. Above $70 \mathrm{~Hz}$, approximately in between the two Nyquist frequencies, the performance of the methods degrades as the grid of measurement microphones and loudspeakers is too sparse to sufficiently measure and compensate for the reflections.

\section{DISCUSSION}

All three separation techniques result in similar sound fields in the experiment. PWD has the advantage of the simplest mathematical formulation. The implementation of the ESM technique is more complex due to the freedom of choices over the positions of the equivalent sources. Intuitively, one could imagine that this is useful in the modeling of reflections and scattering from non-planar surfaces, i.e., the positions of the equivalent sources follow the surface geometry. ${ }^{24}$ However, separate numerical investigations with non-planar back walls (not included here) indicate that the placement of equivalent sources along these surfaces does not lead to increased performance and the planar distribution of the equivalent sources is generally applicable. The SFT technique implicitly assumes either a sound field that is periodic over the sampling region or vanishing pressure near the boundaries. The walls in the rectangular room can be thought of as such a periodic system and the SFT technique therefore performs on par with the others in this geometry. However, in the setup with slanted ceilings (i.e., non-

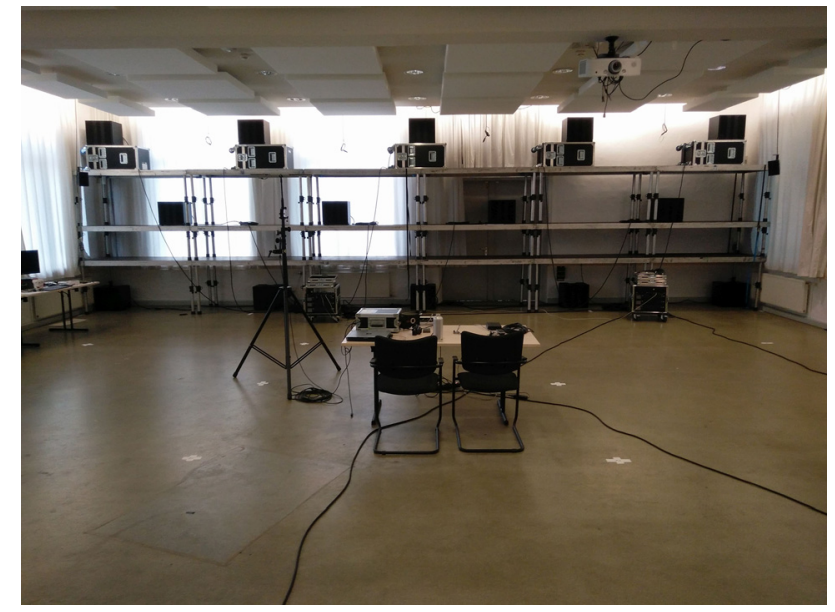

FIG. 6. (Color online) Experimental setup: realization of the secondary subwoofer array using a shelf built out of stage podia.

separable geometry) both assumptions are incorrect and SFT is not able to reduce the room resonances in the same way as the other techniques.

Equation (15) defines the optimal filter for the SFT technique as the minimizer of the reflected absolute squared pressure integrated over the whole space of wavenumbers. This representation makes it also possible to only reduce the propagating plane wave components with real wavenumber $k_{z}$ by changing the integration domain to the radiation disk, $k_{x}^{2}+k_{y}^{2} \leq k_{z}^{2}$. While doing so nearly completely removes the radiating wave components in the SFT representation, it also increases the evanescent components, which then dominate the sound field in an uncontrolled way.

The active compensation system is reducing the sound pressure at the resonance frequencies in the numerical and experimental studies (Figs. 4, 5, and 7) in the whole room compared to the uncontrolled cases. Why does the sound pressure not increase, although many more loudspeakers are drawing electrical power and are contributing to the sound field at these frequencies? Where does this energy go? Nelson $^{25}$ highlights that the mechanism of active sound absorption is a negative acoustical power radiated by the loudspeaker. The sound field is supporting the movement of the loudspeaker such that less electrical input power is needed to drive it.

CABS was experimentally implemented with more success in rooms with typical living room dimensions and only two loudspeakers per side. ${ }^{12,13}$ We assume that the bad performance of the present CABS implementation is related to the larger room size and deviations from a perfectly rectangular room (furniture and window and door recesses as seen in Fig. 6). To have a plane wave sound field up to the same frequency, but in a larger room, one has to position the loudspeakers at the nodal planes of modes with higher order, e.g., $(0,4,1)$ instead of $(0,2,1)$. However, these higher order nodal planes will also vary more strongly from their theoretical positions in a perfectly rectangular room due to the imperfections of the real room geometry, leading to the excitation of modes different from the $x$ axial modes. The scalability of CABS is therefore restricted by both the number of available loudspeakers and the size of the room. 
single responses — mean - - - STD - *- phase
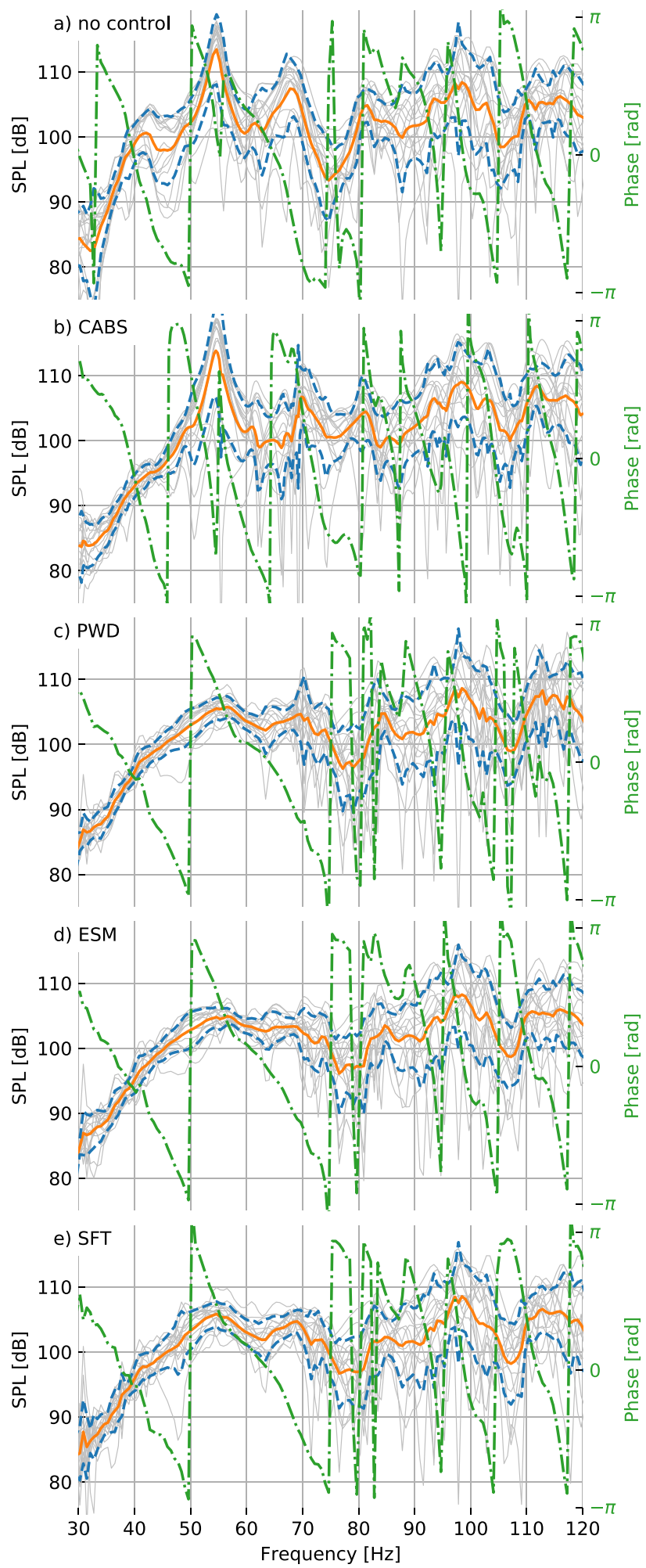

FIG. 7. (Color online) Experimental room frequency responses for the different control techniques. Single magnitude responses (thin line), their average (continuous line), and standard deviation (dashed line). The phase response is given for one position in the center of the room (dashed-dotted line).

In contrast, the reproduction method proposed here is applicable to any room size and geometry. However, a somewhat large number of loudspeakers and microphones is needed.
For the application of the method in sound reinforcement, care has to be taken to only drive the secondary sources in the frequency range, where they behave as absorbers. Otherwise, the secondary sources will introduce audible artifacts. Future research should investigate the frequency limits depending on the density of secondary sources and microphones in the double layer array and implement the method for real-time application.

\section{CONCLUSIONS}

This study has investigated how the low frequency response in rooms can be controlled using an array of secondary sources that absorbs incident sound created by the primary sound system. The signals to the secondary sources are calculated by separation of the sound field into incident and reflected components using a microphone array and the minimization of the reflections. The method is applicable to arbitrary room geometries and can globally improve the sound field at low frequencies by reducing room resonances.

Three different sound field separation techniques have been investigated, which perform similarly in the case of a rectangular room. The plane-wave decomposition technique gave the best results for the more complicated room geometry in the numerical study while also being the simplest to implement. An investigation of the PWD, SFT, and ESM techniques showed that sound field separation as a tool for active room compensation can be successfully implemented in multiple ways.

\section{ACKNOWLEDGMENTS}

We kindly thank Felix Einsiedel, Simon Heinze, and Matthias Christner and the many others at $\mathrm{d} \& \mathrm{~b}$ audiotechnik for insightful discussions, for their helping hands in the experiments, and for the supply of equipment and facilities.

${ }^{1}$ N. W. Adelman-Larsen, E. R. Thompson, and A. C. Gade, "Suitable reverberation times for halls for rock and pop music," J. Acoust. Soc. Am. 127(1), 247-255 (2010).

${ }^{2}$ S. J. Elliott and P. A. Nelson, "Multiple-point equalization in a room using adaptive digital filters,” J. Audio Eng. Soc. 37(11), 899-907 (1989).

${ }^{3}$ P. A. Nelson, F. Orduña-Bustamante, and H. Hamada, "Inverse filter design and equalization zones in multichannel sound reproduction," IEEE Trans. Audio, Speech, Lang. Process. 3(3), 185-192 (1995).

${ }^{4}$ A. Mäkivirta, P. Antsalo, M. Karjalainen, and V. Välimäki, "Modal equalization of loudspeaker-Room responses at low frequencies," J. Audio Eng. Soc. 51(5), 324-343 (2003).

${ }^{5}$ M. Furstoss, D. Thenail, and M. A. Galland, "Surface impedance control for sound absorption: Direct and hybrid passive /active strategies," J. Sound. Vib. 203(2), 219-236 (1997).

${ }^{6}$ H. Lissek, R. Boulandet, and R. Fleury, "Electroacoustic absorbers: Bridging the gap between shunt loudspeakers and active sound absorption," J. Acoust. Soc. Am. 129(5), 2968-2978 (2011).

${ }^{7}$ M. D. F. V. Pereira, A. M. Pasqual, and G. D. S. Papini, "Numerical and theoretical analysis of sound absorption by an actively controlled electrodynamic loudspeaker," J. Braz. Soc. Mech. Sci. Eng. 39(1), 81-87 (2017).

${ }^{8}$ E. Rivet, S. Karkar, and H. Lissek, "Broadband low-frequency electroacoustic absorbers through hybrid sensor-/shunt-based impedance control," IEEE Trans. Control Syst. Technol. 25(1), 63-72 (2017).

${ }^{9}$ A. O. Santillán, "Spatially extended sound equalization in rectangular rooms,” J. Acoust. Soc. Am. 110(4), 1989-1997 (2001). 
${ }^{10}$ A. O. Santillán, C. S. Pedersen, and M. Lydolf, "Experimental implementation of a low-frequency global sound equalization method based on free field propagation," Appl. Acoust. 68(10), 1063-1085 (2007).

${ }^{11}$ C. S. Pedersen and H. Møller, "Sound field control for a low-frequency test facility," in 52nd International AES Conference: Sound Field Control_Engineering and Perception, Audio Engineering Society (2013), pp. 1-10.

${ }^{12}$ A. Celestinos and S. B. Nielsen, "Controlled acoustic bass system (CABS) a method to achieve uniform sound field distribution at low frequencies in rectangular rooms," J. Audio Eng. Soc. 56(11), 915-931 (2008).

${ }^{13}$ J. A. Hargreaves and M. Wankling, "Implementing wave field synthesis in an ITU spec listening room part 2: Bass without modes," in Proceedings of the Reproduced Sound 2011: Sound Systems: Engineering or Art?, Institute of Sound, Brighton, UK (2011).

${ }^{14} \mathrm{~A}$. Celestinos and S. B. Nielsen, "Low frequency sound reproduction in irregular rooms using CABS (Control Acoustic Bass System)," in Proceedings of Forum Acusticum 2011 (European Acoustics Association EAA, Forum Acusticum), pp. 293-298, available at http://vbn.aau.dk/ en/publications/low-frequency-sound-reproduction-in-irregular-roomsusing-cabs-control-acoustic-bass-system(704960f0-f3b6-4543-acb57ad96346eab2).html.

${ }^{15}$ M. Tamura, "Spatial Fourier transform method of measuring reflection coefficients at oblique incidence. I: Theory and numerical examples," J. Acoust. Soc. Am. 88(5), 2259-2264 (1990).
${ }^{16}$ E. Fernandez-Grande and F. Jacobsen, "Sound field separation with a double layer velocity transducer array (L)," J. Acoust. Soc. Am. 130(1), 5-8 (2011).

${ }^{17}$ E. Fernandez-Grande, F. Jacobsen, and Q. Leclère, "Sound field separation with sound pressure and particle velocity measurements," J. Acoust. Soc. Am. 132(6), 3818-3825 (2012).

${ }^{18}$ H. Zhu, R. Rajamani, and K. A. Stelson, "Active control of acoustic reflection, absorption, and transmission using thin panel speakers," J. Acoust. Soc. Am. 113(2), 852-870 (2003).

${ }^{19}$ N. Han, S. Feng, and X. Qiu, “Active control of one-dimension impulsive reflection based on a prediction method," J. Acoust. Soc. Am. 127(3), 1193-1196 (2010).

${ }^{20} \mathrm{M}$. Norambuena, "Mathematical model for a multichannel active absorption system," Acta Acust. Acust. 99(6), 905-916 (2013).

${ }^{21}$ E. G. Williams, Fourier Acoustics: Sound Radiation and Nearfield Acoustical Holography (Academic Press, San Diego, CA, 1999), pp. 107-112.

${ }^{22}$ G. H. Golub, M. Heath, and G. Wahba, "Generalized cross-validation as a method for choosing a good ridge parameter," Technometrics 21(2), 215-223 (1979).

${ }^{23}$ Y. Miki, "Acoustical properties of porous materials-Modifications of Delany-Bazley models,” J. Acoust. Soc. Jpn. 11(1), 19-24 (1990).

${ }^{24}$ G. H. Koopmann, L. Song, and J. B. Fahnline, "A method for computing acoustic fields based on the principle of wave superposition," J. Acoust. Soc. Am. 86(6), 2433-2438 (1989).

${ }^{25}$ P. A. Nelson, Active Control of Sound (Academic Press, London, 1992), pp. 140-142. 